\title{
ENERGÍA DE BAJA INTENSIDAD: gobiernos, mercados e instituciones en el regionalismo energético de América del Sur
}

\author{
ENERGIA DE BAIXA INTENSIDADE: governos, mercados e \\ instituições no regionalismo energético de América do Sul
}

\author{
Stefano Palestini*
}

\begin{abstract}
A pesar de haber sido considerada un área prioritaria de cooperación, el regionalismo energético sudamericano muestra signos de estancamiento, evidenciados por las infructuosas negociaciones de un Tratado Energético Sudamericano. Este artículo explica la paradoja de por qué, no obstante la voluntad política, las favorables condiciones estructurales para la integración, y la creación de instituciones regionales ad hoc, el regionalismo energético no consiguió pasar de las declaraciones intergubernamentales a la implementación de políticas. Se argumenta que ninguna de las instituciones creadas ha sido capaz de mediar la divergencia entre mercados y entre preferencias gubernamentales. Se requiere por tanto de un nuevo consenso que replantee la necesidad y la capacidad de la región para llevar adelante la agenda de integración energética. A un nivel más general, el caso del sector energético brinda lecciones importantes acerca de los desafíos futuros que enfrenta la cooperación regional en materia de políticas públicas en Sudamérica.

Palabras-CLAVEs: Regionalismo. América del Sur. Integración energética. UNASUR-IIRSA.
\end{abstract}

\section{INTRODUCCIÓN}

Sudamérica es una región favorecida en términos de la dotación de recursos energéticos. Contiene el 19\% de las reservas globales de petróleo, y el 3,44\% de las reservas globales de gas. Su potencial hidroeléctrico, concentrado en la cuenca de Amazonas y del Plata, excede los 600 GW, concentrando el 32\% de los recursos hídricos mundiales. Los recursos energéticos se encuentras, además, distribuidos de manera heterogénea entre los países que componen la región: mientras algunos países son importantes productores de hidrocarburos, otros poseen un gran potencial de hidroelectricidad además de ciclos hídricos diferenciados que hacen que estaciones secas en un país correspondan con estaciones lluviosas en otro. En definitiva, la combinación de alta dotación de recursos, por un lado, y de una matriz diferenciada, por otro, hacen de Sudamérica una región estructuralmente propicia para la integración energética regional (Barrera-Hernández, 2012; Organización Latino-

* Universidade Livre de Berlim. Departamento de Ciências Sociais e Políticas, Alemania.

Ihnestraße 26, 14195 Berlin. stefano.palestini@eui.eu americana de Energía (OLADE), 2013; Banco de Desarrollo de América Latina (CAF), 2013).

A la luz de estos factores estructurales, la integración regional energética es una estrategia ganadora para todas las partes. En teoría, la integración regional de las cadenas productivas y de distribución de energía incrementa tanto la seguridad y la eficiencia energética de aquellos países con una baja dotación de recursos - tales como Chile o Uruguay -, como de aquéllos con una matriz energética poco diversificada - tales como Ecuador o Bolivia. La integración energética con los países vecinos es además una opción tanto para los países importadores netos, como también para los exportadores que buscan diversificar los mercados finales para insertar sus excedentes de producción.

Las elites políticas sudamericanas parecen ser conscientes tanto de las condiciones naturales que ofrece la región para integrar los sectores energéticos, como de los beneficios que trae consigo esta política. En efecto, los dos esquemas subregionales - la Comunidad Andina de Naciones, CAN, y el Mercado Común del Sur, MERCOSUR - han elaborado políticas y planes de acción para integrar sus 
mercados energéticos (CAF, 2013). De modo aún más claro, durante la histórica primera Cumbre de Jefes y Jefas de Estado de Sudamérica, en Brasilia el año 2000, los líderes de la región acordaron que la integración energética, junto con la integración de la infraestructura de transporte y telecomunicaciones, serían las áreas prioritarias de un nuevo proyecto de regionalismo a escala sudamericana (Palestini; Agostinis, 2015).

Sin embargo, a pesar de las condiciones favorables y de la aparente voluntad política, a quince años del lanzamiento de la iniciativa los resultados en materia de integración energética son decepcionantes, limitándose a proyectos de interconexión de infraestructura y a acuerdos de cooperación bilateral en desconexión el uno del otro. A la fecha, Sudamérica luce más como un espacio fragmentado de economías nacionales orientadas a lograr su propia seguridad energética, más que al espacio integrado que la Declaración de Brasilia se proponía. Este artículo busca explicar esta brecha entre los dichos y los hechos en el área de la integración energética la cual - prima facie - no deja de ser sorprendente pues, las declaraciones presidenciales han ido acompañadas de la construcción de instituciones regionales con el objetivo de llevar a cabo la integración física y regulatoria de los sectores energéticos nacionales: Integración de la Infraestructura Regional Sudamericana (IIRSA), PETROAMERICA y el Consejo Energético Sudamericano (UNASUR) son ejemplos. ¿Por qué a pesar de condiciones estructurales propicias, de la buena voluntad de los gobiernos, y de la creación de arreglos institucionales ad hoc a nivel regional, el regionalismo energético en Sudamérica parece ser más una utopía que una realidad?

La respuesta que ofrezco en este artículo se basa en nociones básicas de economía política, pero que sin embargo son muchas veces ignoradas a la hora de explicar y evaluar el regionalismo. El regionalismo energético, entendido como un proyecto político orientado a generar un mercado energético regional a través de cooperación e integración, depende de la interacción de tres componentes: los mercados, las preferencias gubernamentales y las instituciones regionales. Esta interacción está doblemente tensionada. Primero, existe una tensión entre el mercado y los gobiernos. La lógica del mercado, es decir las estrategias corporativas de las empresas productoras y de servicios energéticos, no siempre es convergente con la lógica política expresada en las políticas nacionales de energía. En efecto, la mayoría de las veces las dos lógicas no coinciden. De modo general las empresas buscan, por una parte, mayor autonomía y procuran explotar la regulación nacional a favor de la estrategia de negocio, mientras que los gobiernos, por otra parte, poseen grandes incentivos para aumentar los niveles de control sobre la producción de energía, un recurso esencial para sostener cualquier programa de desarrollo económico. El incentivo de sacrificar "eficiencia" por ganar "mayor control" se incrementa cuanto más dependiente es una economía nacional de la producción de recursos energéticos, como es el caso de varios países sudamericanos (ver Aalto, 2015; Belyi; Makarychev, 2015; Talus, 2013).

Segundo, existe una tensión inter-gubernamental. Los recursos energéticos son esenciales para los gobiernos y, por tanto, la seguridad energética es un objetivo nacional prioritario que fácilmente puede entrar en contradicción con la cooperación y, por cierto, aún más con la integración energética que supone un nivel considerable de delegación de competencias a organizaciones regionales (Palestini, 2012; Malamud, 2008). Los gobernantes saben que la integración - a través de infraestructura y de regulación - implica mayor interdependencia entre Estados, y saben que la interdependencia puede traducirse en vulnerabilidad si no existen reglas claras o si los vecinos no parecen ser particularmente dados a respetarlas.

Tercero, las instituciones regionales median estas dos tensiones. Pero, a diferencia de muchos enfoques (neo) institucionalistas que pecan de un optimismo ingenuo, la mediación 
institucional no siempre resuelve los problemas de coordinación y cooperación entre gobierno y mercado, y de gobiernos entre ellos. En efecto, las instituciones regionales pueden ser irrelevantes generando un regionalismo energético débil donde las tensiones anteriormente mencionadas se resolverán a favor del actor más fuerte, sea de mercado (grandes corporaciones) o de un gobierno (el estado más poderoso). Las instituciones regionales pueden también acentuar la tensión, si además de carecer de capacidades son percibidas por los actores como sesgadas a favor de los intereses de uno de los actores, o incluso "captivas" por dichos actores.

El argumento de este artículo es que el regionalismo energético sudamericano ha generado instituciones regionales de baja capacidad (como IIRSA) e instituciones regionales "captivas" (PETROAMERICA, y hasta cierto punto el Consejo Energético de UNASUR) siendo incapaces de resolver las dos tensiones arriba descritas. Los datos que informan este trabajo se basan en entrevistas semi-estructuradas realizadas con autoridades de gobierno, funcionarios de las agencias gubernamentales de energía, funcionarios de bancos regionales de desarrollo (Banco Interamericano de Desarrollo - BID y CAF), académicos e investigadores en centros de política pública energética, además del análisis de documentos oficiales y prensa.

Voy a desarrollar este argumento en tres secciones, más una conclusión. La primera sección describe la tensión existente entre mercados nacionales energéticos que se han desarrollado a través de trayectorias divergentes. Grosso modo, ya a la mitad de la década pasada era posible distinguir, desde el punto de vista de sus sectores energéticos, economías capitalistas de estado (Venezuela, Bolivia, Ecuador), una serie de economías intermedias (Argentina, Brasil, Paraguay, Guyana y Uruguay), y economías liberales de mercado dependientes de la trayectoria de liberalización iniciada a finales de 1980 (Chile, Colombia, Perú). El regionalismo energético sudamericano no ha tomado seriamente en consideración esta divergencia, así como los desafíos que plantea para un proyecto de integración.

La segunda sección analiza la evolución de las preferencias gubernamentales en relación al regionalismo energético. En esta sección analizo las distintas declaraciones intergubernamentales generadas en las cumbres presidenciales de la Comunidad Sudamericana de Naciones y de la UNASUR, que muestran por un lado una creciente divergencia entre las preferencias de los gobiernos que, por una parte, persiguen políticas de capitalismo de estado, y, por otra, de los gobiernos que adhieren a economías liberales de mercado.

La tercera sección analiza las instituciones regionales generadas por mandato de los gobiernos nacionales. La primera de ellas, IIRSA, poseía un diseño institucional innovador que, empero, se demostró rápidamente incapaz de mediar entre los mercados y los gobiernos, así como de resolver las diferencias gubernamentales. A mediados de la década pasada, IIRSA es desplazada por una segunda institución, PETROAMÉRICA echa a la medida de los intereses nacionales de un gobierno (Venezuela). La tercera institución - el Consejo Energético Sudamericano - resulta ser una solución de compromiso que, por una parte, intentó evitar la total desafección de algunos gobiernos respecto al proyecto regional y que, por otra, establece límites estrictos a Venezuela a través de un diseño rígidamente intergubernamental.

El artículo cierra con algunas conclusiones acerca de qué tipo de regionalismo energético caracteriza Sudamérica hoy en día. Como es de sospechar, mi visión no es muy positiva. La elite política sudamericana debe volver a plantearse - como lo hiciera en el 2000 - por qué es deseable la integración energética regional, para sólo entonces enfrentar el gran desafío de una nueva estrategia regional sobre la base de lo aprendido en las experiencias fracasadas anteriores. 


\section{MERCADOS ENERGÉTICOS: trayectorias divergentes}

Los mercados no surgen espontáneamente, sino que son el resultado de la interacción histórica de intereses materiales y de normas; estas últimas regulan el modo en que los intereses se realizan en determinados ámbitos de la actividad económica (Fligstein, 1996; Beckert, 2010). Hablar de los mercados energéticos sudamericanos es ya, de por sí, una simplificación, pues en efecto los mercados presentan variaciones dependiendo de los sub-sectores energéticos y de los distintos eslabones en la cadena de producción de valor. En esta sección abusaré de la simplificación y me referiré a "mercados energéticos" entendiendo por ellos principalmente los sectores de hidrocarburos - gas y petróleo - y eléctrico, en los ámbitos de producción y distribución. Temporalmente me limitaré al período en cuestión, es decir desde la Primera Cumbre de Presidentes de Sudamérica, 2000 hasta el momento actual, 2015.

Como es sabido, las economías latinoamericanas - comprendidas las sudamericanas - experimentaron durante la década de 1990 reformas orientadas a disminuir el control público sobre diversos mercados, acercándolos al modelo ideal de competencia perfecta. El mercado energético, junto con el de la 亏े infraestructura y las telecomunicaciones, son ริำ los ejemplos más evidentes de estas reformas compuestas de tres componentes básicos: la ¿ liberalización de la competencia, la privatiz ización de los activos públicos y la reforma regulatoria (Lutz, 2001; Pollitt, 2012).

A través de la privatización de compañías públicas y de la desintegración de las cadenas productivas verticales, los gobiernos sudamericanos neoliberales intentaron implementar mercados competitivos en los distintos eslabones de la cadena (Rudnick; Zolezzi, 2001; Talus, 2013). Mientras que en Europa la liberalización de los mercados en- ergéticos nacionales tuvo como objetivo la integración de un mercado energético regional, en América Latina la liberalización se orientó a atraer empresas transnacionales que vieron en el mercado Centro y Sudamericano mejores oportunidades de negocio que aquellas de sus propios mercados de origen. A lo largo de los '90s, empresas eléctricas europeas (principalmente españolas), se instalan en América Latina buscando un mercado más seguro que el crecientemente competitivo mercado único Europeo luego de la adopción de la Directiva 96/92 en 1996 (Rozas, 2009; Trillas; Montoya, 2011; Lutz, 2001). También en el sector de hidrocarburos, empresas tales como Shell, Repsol y Total expandieron sus operaciones adquiriendo activos públicos, asociándose con empresas públicas y/o controlando holdings (Kozulj, 2008). A finales de la década de 1990, la mayoría de las economías sudamericanas correspondían - desde el punto de vista de sus mercados energéticos - a economías liberales de mercado, con un alto nivel de transnacionalización. Incluso grandes empresas públicas tales como Petróleos de Venezuela S.A (PdVSA), en Venezuela, y Petrobras, en Brasil, experimentaron privatizaciones parciales.

En 1998, la elección del Presidente Hugo Chávez inicia una sucesión de victorias electorales de partidos y coaliciones de izquierda con programas críticos de las ideas que inspiraron las reformas de mercado de la década de 1990. Como alternativa al neoliberalismo, los gobiernos de izquierda proponen programas basados en profundas reformas políticas y sociales para cuya realización requieren de un Estado que sea capaz de apropiarse de la renta de los recursos naturales - en particular de los recursos energéticos - para su re-utilización en las iniciativas de transformación social. El gobierno de Hugo Chávez en Venezuela, el gobierno de Evo Morales en Bolivia, el gobierno de Néstor Kirchner y Cristina Fernández Kirchner en Argentina, y el gobierno de Rafael Correa en Ecuador persiguen - con profundidades variables - una política de economía capitalista de 
Estado en el sector energético. A diferencia del capitalismo liberal de mercado que se basa en la generación de riqueza a través de la competencia de agentes privados, el capitalismo de Estado se basa en la generación de riqueza a través de Empresas Públicas protegidas y favorecidas (campeones nacionales) y de la apropiación y re-utilización del excedente por parte del Estado (Ostrowski, 2015).

Aun cuando todas las Constituciones nacionales de los países sudamericanos confieren al Estado la propiedad de los recursos, hacia la mitad de los 2000 las economías sudamericanas muestran ya una marcada diversidad en los modelos de control sobre las diferentes actividades de la cadena de valor energética. El Cuadro 1 muestra la estructura de los sectores energéticos nacionales (para estos efectos, el sector de hidrocarburos y eléctrico) de acuerdo al grado de control que el Estado ejerce sobre la cadena de valor. "Alto control" significa que el ordenamiento jurídico nacional confiere al Estado el control total de las actividades. "Control medio" significa que el ordenamiento jurídico nacional confiere al Estado control, pero permite la participación privada. "Bajo control" significa que el ordenamiento jurídico nacional reserva un mínimo de control al Estado, y una participación mayoritaria al sector privado. Estas diferencias se traducen en modalidades diferentes de contratos, royalties, impuestos y tipos de agentes reguladores (OLADE, 2013).

Cuadro 1 - Nivel de control estatal sobre la cadena de valor en los sectores eléctrica y de hidrocarburos

\begin{tabular}{|l|l|l|}
\hline Nivel de control & \multicolumn{1}{|c|}{ Sector Eléctrico } & \multicolumn{1}{|c|}{ Sector Hidrocarburos } \\
\hline Alto control & Venezuela, Surinam, & Venezuela, Surinam, \\
& Ecuador, Bolivia, Paraguay & Ecuador, Bolivia \\
Control medio & Argentina, Brazil, Guyana, & Argentina, Brazil, Chile, \\
& Uruguay & Guyana Paraguay, Uruguay \\
Bajo control & Chile, Colombia, Perú & Colombia, Guyana, Perú \\
\hline
\end{tabular}

Fontes: Elaboración propia a partir de datos de OLADE (2013).

La divergencia entre los sectores energéticos nacionales que era ya evidente a mediados de los 2000 impone un fuerte desafío a un proyecto de integración regional. Primero, demuestra que los gobiernos de la región han resuelto la tensión entre mercado/Estado de modo radicalmente diferente. Países tales como Chile, Colombia y Perú han continuado la trayectoria iniciada con las liberalizaciones de 1990, mientras Bolivia, Ecuador y Venezuela se acercan al modelo de Capitalismo de Estado anteriormente descrito. Segundo, implica que los gobiernos de la región tienen márgenes de agencia considerablemente diferentes para implementar políticas de cooperación e integración tales como redes de infraestructura y armonización regulatoria. Finalmente, implica que las preferencias gubernamentales serán divergentes a la hora de negociar instrumentos de cooperación e integración regional.

\section{EVOLUCIÓN DE LAS PREFEREN- CIAS GUBERNAMENTALES}

Los gobiernos sudamericanos decidieron embarcarse en un proyecto de regionalismo energético cuando el proceso de divergencia entre economías liberales de mercado y capitalismos de Estado - descrito en la sección anterior - aún no se iniciaba. En esta sección describiré la evolución de las preferencias gubernamentales en relación al regionalismo energético a través del análisis de las Declaraciones intergubernamentales partiendo desde la primera Cumbre de Jefes y Jefas de Estado de Sudamérica, en Brasilia 2000, lugar en el que el proyecto de regionalismo sudamericano fue oficialmente lanzado por gobiernos alineados en su gran mayoría con las ideas que inspiraron las reformas neoliberales de mercado.

La Primera Cumbre de Presidentes Sudamericanos fue un hecho histórico pues por primera vez Sudamérica asumía un proyecto colectivo separado de Centro América y de México. Los factores que explican el origen de esta Cumbre 
son complejos y obedecen a cálculos geopolíticos fundamentalmente de la diplomacia brasilera, así como a la particular coyuntura económica que vivía la región (Palestini; Agostinis, 2015). Para los efectos de este artículo, interesa destacar que el principal resultado de la Cumbre fue la creación de una nueva institución regional - la Iniciativa para la Integración de la Infraestructura Regional Sudamericana, o IIRSA - con el fin de integrar energéticamente a los países de la región (además de integrar la infraestructura de transporte y telecomunicaciones). La Cumbre fue exitosa precisamente porque logró generar un consenso básico entre los doce gobiernos en torno a una iniciativa concreta en áreas de alto interés - energía y transporte - y que además los presidentes percibían como menos complejas políticamente, en comparación con el comercio o seguridad.

La integración energética se reforzó en la agenda del naciente regionalismo sudamericano durante la segunda Cumbre de Presidentes en Guayaquil 2002, en gran parte por la relevancia que el país anfitrión - Ecuador - le daba al sector energético. La Declaración de Guayaquil (también conocido como el "Consenso de Guayaquil”) enfatiza no sólo la integración de la infraestructura energética, sino también la armonización de la regulación de los mercados energéticos ${ }^{1} \mathrm{e}$ incluso establece el manda• to para que IIRSA elabore una Carta Energéti$\stackrel{\circ}{\circ}$ ca Sudamericana. ${ }^{2}$ En 2004, en la Cumbre de ขึ Cusco, la integración energética en el marco de IIRSA continúa siendo un elemento central del - proyecto regionalista bautizado como: Comu\% nidad de Naciones Sudamericanas (Palestini; Agostinis, 2014; Sanahuja, 2012).

Un año más tarde, sin embargo, la agenda energética inicia a cambiar con la realización de la Primera Cumbre de Ministros de Energía en Caracas, que antecede y prepara la agenda de la Cumbre Presidencial de Brasilia en Septiembre de 2005. Durante estas dos cumbres, el ejecutivo Venezolano a través de su canciller, Alí Rodríguez, y del propio presidente Chávez, dieron señales claras de que la integración energética regional era un objetivo prioritario para el país y que estarían dispuestos a liderar el proceso. Sin embargo el liderazgo venezolano no se realizaría a través de IIRSA, sino a través de una nueva institución, PETROAMÉRICA, y sus sub-programas PETROSUR y PETROANDINA. A pesar que la cumbre presidencial tuvo lugar en la capital Brasilera, la agenda de la cumbre y su declaración reflejan explícitamente el programa Venezolano, avalado por los gobiernos de la región con la excepción de los presidentes de Colombia, Paraguay y Uruguay que no atendieron la reunión. La declaración prácticamente no menciona IIRSA, y la agenda energética pasa íntegramente a ser parte de PETROAMERICA, siendo por primera vez separada de la agenda de integración de infraestructura (Couto; Padula, 2012). ${ }^{3}$

Las Declaraciones de Caracas y de Brasilia 2005, reflejan además tensiones intergubernamentales en relación al sector energético. Por ejemplo, en su Preámbulo se considera que PETROAEMERICA no se limita al sector de los hidrocarburos, sino que al sector energético en su totalidad, ${ }^{4}$ lo cual puede interpretarse como una salvaguarda al predominio de las iniciativas de cooperación petroleras auspiciadas por Venezuela a través de su empresa pública PdVSA. La siguiente Cumbre de Cochabamba, 2006, es nuevamente dominada por la agenda Venezolana. A pesar de ser respaldada por la mayoría de los países sudamericanos, IIRSA es fuertemente cuestionada por los presidentes Chávez, el anfitrión Evo Morales y el presidente electo Rafael Correa, por su carácter "extractivista” y financiero, y no es mencionada en absoluto en la declaración final, intitulada "Un Nuevo Modelo de Integración del Siglo XXI" (Palestini; Agostinis, 2015).

\footnotetext{
${ }^{3}$ Declaración de Brasilia 2005, párrafo 17.

${ }^{4}$ Declaración de Caracas 2005, Preámbulo.
} 
La polarización entre los gobiernos llega a su nivel más alto en la Primera Cumbre Energética Sudamericana, en Isla Margarita (2007), reunión en la que se crea la UNASUR y el Consejo Energético Sudamericano. ${ }^{5}$ Aunque la creación de estas dos instituciones se puede considerar una victoria para el proyecto venezolano de regionalismo energético, la verdad es que la declaración presidencial, breve y vaga en su contenido, revela más bien una falta de apoyo intergubernamental a las nuevas iniciativas creadas. IIRSA no es mencionada, pero tampoco PETROAMERICA ni el nuevo Consejo Energético ocupan un lugar central en la declaración. El Banco del Sur, otra iniciativa central en el regionalismo Venezolano, ni siquiera entra en la agenda de discusión de la cumbre, gracias a los esfuerzos diplomáticos brasileros que querían evitar mayores tensiones durante la cumbre. Venezuela asume la Presidencia Pro-tempore del Consejo Energético y propone plazos para la elaboración de un Tratado Energético Sudamericano, plazos que son objetados por el gobierno brasilero que propone aplazar la discusión del Tratado sin dar fechas para su firma por los presidentes y, más encima, propone fragmentar su contenido en temas de acuerdo a los intereses nacionales de cada país, lo cual puede fácilmente ser interpretado como un intento por diluir la iniciativa.

En mi interpretación, la Cumbre de Isla Margarita (paradójicamente conocida como la "Primera Cumbre Energética”), marca el inicio de la disolución del regionalismo energético sudamericano. La cumbre, de hecho fue precedida por una serie de tensiones bilaterales relacionadas con el sector energético y que directa o indirectamente involucraron a los gobiernos venezolano y brasilero. La poco mesurada implementación de la Ley de hidrocarburos por parte del gobierno del Presidente Morales en Bolivia ocasionó no solo pérdidas a Petrobras,

${ }^{5}$ UNASUR (Unión de Naciones Sudamericanas) fue el nombre que reemplazó a la "Comunidad Sudamericana de Naciones". El gobierno Venezolano venía desde hace algún tiempo abogando por el cambio de nombre de "Comunidad" a "Unión". que por la época controlaba $46 \%$ de las reservas de gas boliviano, sino que por sobre todo generó fuertes presiones internas al gobierno del Presidente Lula las que se intensificaron cuando se comunicó que el gobierno Venezolano había ofrecido apoyo financiero y técnico para llevar a cabo la reforma en Bolivia. Contemporáneamente, el presidente Chávez había emitido abiertas y vociferantes críticas en contra del acuerdo firmado entre Brasil y los Estados Unidos para el desarrollo de la industria de biocombustibles, que constituye un competidor directo del combustible fósil (Oliveira, 2008; Manduca, 2012; Philipp; Panizza, 2011).

Estas tensiones intergubernamentales contribuyeron a que la diplomacia Brasilera promoviera una solución de compromiso, encapsulando las iniciativas de PETROAMERICA dentro de un Consejo Ministerial (el Consejo Energético), una estructura con un proceso de toma-de decisiones rígido que requiere no sólo del consenso de los doce ministros de energía, sino del consenso de los doce presidentes para poder llevar adelante cualquier iniciativa por limitada que sea esta.

El Cuadro 2 intenta captar la variación de las preferencias gubernamentales por un programa de integración energética, a partir de las declaraciones intergubernamentales y entrevistas con actores que participaron en las respectivas negociaciones. La segunda columna esquematiza las preferencias de los gobiernos al momento de la Primera Cumbre de Presidentes Sudamericanos en Brasilia (2000) cuando IIRSA es creada, y la tercera columna representa las preferencias al momento de la Primera Cumbre Energética Sudamericana en Isla Margarita (2007), cuando PETROAMERICA es discutida y cuando es creado el Consejo Energético.

Como es posible apreciar en el Cuadro 2, las preferencias al momento de la Cumbre de Isla Margarita mostraban que el consenso original que había permitido lanzar un proyecto de regionalismo energético, estaba fuertemente erosionado. Esto se traducirá en el diseño de las instituciones regionales. 
Cuadro 2 - Intensidad de las Preferencias por un Programa de Integración Energética 2000 y 2007

\begin{tabular}{|c|c|c|}
\hline Gobierno & Preferencias por IIRSA (2000) & $\begin{array}{c}\text { Preferencias por } \\
\text { PETROAMERICA (2007) } \\
\end{array}$ \\
\hline Brasil & $\begin{array}{l}\text { Alta. Reforzar los lazos regionales, mientras } \\
\text { la agenda de integración comercial permanecía } \\
\text { bloqueada. }\end{array}$ & $\begin{array}{l}\text { Baja. Excluye la producción de biocombustibles. } \\
\text { La posibilidad de joint ventures entre Petrobras } \\
\text { y PdVSA es vista como instrumental para } \\
\text { mantener relaciones con Venezuela. }\end{array}$ \\
\hline $\begin{array}{l}\text { MERCOSUR } \\
\text { (Argentina, } \\
\text { Paraguay and } \\
\text { Uruguay) }\end{array}$ & $\begin{array}{l}\text { Alta. Expandir mercados para los excedentes } \\
\text { eléctricos (Paraguay); mejorar la seguridad } \\
\text { energética (Uruguay y Argentina) }\end{array}$ & $\begin{array}{l}\text { Media. Posibilidades de interconexión gasífera e } \\
\text { intercambios petrolíferos a precios favorables } \\
\text { a través de PETROSUR. }\end{array}$ \\
\hline Chile & Alta. Mejorar la seguridad energética. & $\begin{array}{l}\text { Baja. Beneficios inciertos. Es percibida como una } \\
\text { iniciativa que compite con IIRSA y con el proyecto } \\
\text { del Anillo Energético. }\end{array}$ \\
\hline Perú & $\begin{array}{l}\text { Alta. Expandir los mercados para los excedentes } \\
\text { energéticos. }\end{array}$ & $\begin{array}{l}\text { Baja. Beneficios inciertos. Es percibida como una } \\
\text { iniciativa que compite con IIRSA. Conflictos entre } \\
\text { el ejecutivo peruano y venezolano. }\end{array}$ \\
\hline Bolivia & $\begin{array}{l}\text { Alta. Expandir los mercados para los excedentes } \\
\text { de la producción de gas natural. }\end{array}$ & $\begin{array}{l}\text { Alta. Posibilidades de jointventure con PdVSA e } \\
\text { intercambios petrolíferos a precios convenientes } \\
\text { a través de PETROANDINA. }\end{array}$ \\
\hline Ecuador & Alta. Diversificar la matriz energética. & $\begin{array}{l}\text { Alta. Posibilidades de joint venture con PdVSA e } \\
\text { intercambios petrolíferos a precios convenientes } \\
\text { a través de PETROANDINA. }\end{array}$ \\
\hline Colombia & Alta. Diversificar la matriz energética. & $\begin{array}{l}\text { Baja. Beneficios inciertos. Conflictos } \\
\text { intergubernamentales con Venezuela. }\end{array}$ \\
\hline
\end{tabular}

Fonte: Elaboración propia a partir de entrevistas con actores clave.

\section{INSTITUCIONES REGIONALES: IIRSA, Petroamerica y el Consejo Energético Sudamericano}

En esta tercera sección quiero detenerme a analizar el diseño de las instituciones regionales creadas por los gobiernos sudamericanos para la integración energética de la $\stackrel{0}{0}$ región. Como se ha dicho en la Introducción, es un error suponer que las instituciones, por 今ึ sí mismas, resuelven los problemas de coordinación y cooperación en un área de políticas. Tampoco es correcto asumir que la sola creag. का म

\section{EI modelo IIRSA (2000-2006)}

Desde el punto de vista de su diseño institucional, IIRSA fue una iniciativa inno- vadora. Fue creada por mandato de la Primera Cumbre de Brasilia en 2000, como una institución informal sin un tratado constitutivo y sin una burocracia o presupuesto específicos. Sus objetivos y su metodología de planificación original - basada en el concepto de Ejes de Integración y Desarrollo - fue el fruto de la cooperación entre comunidades epistémicas: planificadores brasileros inspirados por la obra de Eliezer Batista bajo los gobiernos de F.H. Cardoso, el Banco Inter-Americano de Desarrollo (BID), y el Banco de Desarrollo de América Latina (CAF). Los Presidentes Sudamericanos acordaron en Brasilia, delegarle la dirección de IIRSA al BID, a la CAF y al pequeño fondo de la cuenca del Plata, FONPLATA que en su conjunto integraron el Comité de Coordinación Técnica de IIRSA (Palestini; Agostinis, 2015; Tavares, 2012).

Gracias a la relativa autonomía que gozaron los bancos regionales durante los primeros seis años de regionalismo sudamericano, los bancos regionales consiguieron dotar a IIRSA de una estructura organizacional básica, 
crear vínculos incipientes entre gobiernos y actores privados, $\mathrm{y}$ - quizás su más importante logro - producir una cartera de proyectos consensuados por los gobiernos y un conjunto de metodologías de planificación para implementarlos (Palestini; Agostinis, 2015; Bruszt; Palestini, 2016; Rubio, 2013).

Sin embargo, las capacidades institucionales de IIRSA se mostraron al poco tiempo limitadas. Los bancos regionales no lograron generar los incentivos necesarios para trasladar los proyectos de integración energética y de transporte del papel a la realidad. Las capacidades de los bancos también resultaron ser limitadas para sorteara los cambios políticos al interior de los países sudamericanos. En efecto, los gobiernos de Venezuela, Ecuador y Bolivia retiraron el apoyo a los bancos y, por consiguiente, a IIRSA cuestionando sus metodologías y sus criterios de selección de proyectos. Como veremos más adelante, tampoco Brasil - normalmente citado por la literatura como el "líder regional" - apoyó a los bancos en su proyecto de llevar adelante una estrategia de integración regulatoria energética; el gobierno brasilero, a través de su Ministerio de Minas y Energías desacopló su política energética nacional del proyecto regional. Desde el 2006 en adelante, IIRSA prosigue sus actividades con un débil mandato gubernamental y con un exclusivo foco en proyectos de integración física de transporte, dejando la agenda energética en un lugar secundario.

\section{El modelo PETROAMERICA (2005-2007)}

La agenda energética es retomada desde el 2005 por PETROAMERICA. A diferencia de IIRSA cuya legitimidad provenía del acuerdo de los doce Jefes de Estado, y su capacidad técnica era brindada por los bancos regionales de desarrollo, PETROAMERICA fue fundamentalmente una iniciativa basada en los objetivos de la política externa venezolana y la capacidad técnica de PdVSA, desde el 2002 bajo el control total del gobierno bolivariano (Pérez; Kfuri, 2011; Serbin, 2011). PETROAMERICA alberga otros tres sub-programas, PETROCARIBE, PETROSUR y PETROANDINA. Al igual que la Alianza Bolivariana para los Pueblos de Nuestra América (ALBA), todas estas iniciativas se basan en el concepto de Compañías Grannacionales, un juego lingüístico respecto a Compañía Transnacional. El concepto es definido por los textos fundacionales del ALBA como "aquéllas corporaciones públicas integradas, en las cuáles la producción se orientará al mercado intra-regional y cuyas operaciones serán llevadas a cabo de manera eficiente" (citado en Pérez y Kfuri, 2011, p. 409). El concepto de Grannacional está, por tanto, íntimamente ligado a un modelo de Capitalismo de Estado; se trata en efecto, de joint ventures entre empresas públicas nacionales orientadas a administrar las redes y cadenas de energía, soslayando los mecanismos de mercado y la armonización regulatoria (Barrera-Hernández, 2012).

El concepto de Grannacional resultaba estratégico para el gobierno venezolano especialmente en el sector de los hidrocarburos. A través de joint ventures con la brasilera Petrobras, Venezuela esperaba suplir el déficit en inversión y de capacidad técnica para explorar, desarrollar y refinar yacimientos de crudo pesado ubicado en la cuenca del Orinoco (Arriagada, 2006). Sin embargo, las iniciativas Grannacionales de PETROAMERICA demostraron ser, a partir de los datos disponibles, experiencias limitadas, si no fallidas. PETROANDINA se tradujo exclusivamente en un memorándum de entendimiento entre Venezuela y Ecuador para refinar petróleo crudo, y en un acuerdo entre PdVSA y la boliviana Yacimientos Petrolíferos Fiscales Bolivianos (YPFB) para suplir petróleo a Bolivia a precios favorables (Gustafson; Elliot, 2011). PETROSUR por su parte incluía proyectos de mayor envergadura. El primer de ellos fue un acuerdo entre PdVSA y Petrobras para construir una refinería en el Estado de Pernambuco - General Abreu Lima. La estructura financiera de esta Grannacional 
se acordó en un $40 \%$ del capital por parte de PdVSA y un $60 \%$ por parte de Petrobras. Sin embargo, a la fecha PdVSA no ha desembolsado ningún activos en el proyecto, el cual se encuentra bajo investigación judicial por parte del Tribunal de Contas da União, en Brasil. El segundo proyecto, aún más ambicioso consistía en la construcción de un gasoducto que conectaría Venezuela, pasaría por la Amazonía brasilera, y fornecería a Argentina y a Uruguay con una potencial extensión a Chile. Ya en 2007 el proyecto, conocido como Gran Gasoducto del Sur, fue considerado técnicamente y económicamente inviable (Palestini; Agostinis, 2015; Arriagada, 2006; Sanahuja, 2012).

PETROAMERICA y sus sub-programas no fueron solamente instituciones con baja capacidad, sino además resultaron ser instituciones desintegradoras y, por ende, contraproducentes desde el punto de vista de un proyecto regionalista. Las razones están a la vista. El concepto jerárquico y centralizado de Grannacional, tenía un fuerte sesgo por economías nacionales organizadas como Capitalismo de Estado, es decir economías donde el gobierno controla las empresas productoras y distribuidoras de energía. La aversión de PETROAMERICA hacia los mecanismos de mercado incluidas las asociaciones público-privadas, excluyó en la práctica a las economías liberales de mercado - Chile, Perú, Colombia - así como a las economías mixtas. El resultado fue que PETROAMERICA solo sobrevivió un par de años en la agenda regional. La así llamada Primera Cumbre Energética Sudamericana, en Isla Margarita en 2007, la reemplazó por un último modelo de institución regional.

\section{EI Consejo Energético Sudamericano de UNASUR (2007 en adelante)}

Como fue dicho más arriba, la Cumbre de Isla Margarita debe ser entendida como un esfuerzo - fundamentalmente de la diplomacia brasilera - por evitar el colapso del proyecto re- gional a través de un acuerdo de mínimos, que pusiera límites y cortapisas al proyecto bolivariano mediante una institucionalidad rígidamente intergubernamental y nulas competencias. Desde esta perspectiva, el Consejo Energético reproduce a escala sectorial, el diseño de UNASUR formalizado en 2008 en su Tratado Constitutivo: formalización sin delegación, parece haber sido la solución de compromiso encontrada por la diplomacia brasilera para imponer un ritmo más pausado a la integración sudamericana sin detenerla totalmente.

El diseño del Consejo Energético obedeció al siguiente cálculo racional: primero, evitar que los gobiernos sudamericanos - particularmente aquellos con Economías Liberales de Mercado - abandonasen el proyecto regional y, segundo, evitar que el proyecto bolivariano - basado en iniciativas tales como PETROAMÉRICA o el Banco del Sur - continuase expandiéndose y erosionando el ya frágil consenso intergubernamental. Siguiendo este cálculo, la diplomacia brasilera optó por un diseño institucional estrictamente intergubernamental. El Consejo es básicamente un foro de ministros de energía que requiere del consenso entre los doce ministros para elevar decisiones que, a su vez, deben ser aceptadas por consenso por los doce Jefes de Estado. Los bancos regionales que formaban el esqueleto y músculo de IIRSA, están ausentes del Consejo Energético. Organizaciones regionales tales como la Organización Latinoamericana de Energía (OLADE) o la CEPAL que podrían brindar visión estratégica y marcos conceptuales de política pública, tienen una baja participación en el consejo que continúa siendo coordinado desde Caracas bajo el mismo concepto de Grannacional que, como se ha argumentado, carece de viabilidad en su implementación por las características heterogéneas de los mercados energéticos nacionales.

Paradójicamente, el Consejo ha asumido el ambicioso objetivo de redactar un Tratado Energético Sudamericano aprobado en 2010 por el Consejo de Jefes de Estado de UNASUR. Luego de dos años sin avances en la ne- 
gociación del Tratado, se creó - a pesar de la oposición del gobierno Brasilero - un Grupo Jurídico-Político separado del Grupo de Expertos con el mandato de preparar un borrador del contenido del Tratado compuesto de siete partes más un preámbulo. El Grupo se ha enfocado en la discusión de los capítulos sobre integración regulatoria, sin lograr un consenso técnico acerca de elementos esenciales tales como el tránsito interno de la energía y el principio de no discriminación. Mientras algunos países entienden el principio de no-discriminación como aplicable únicamente a aquéllos recursos producidos en América del Sur, otros países sostienen que el principio debe aplicarse a todos los recursos energéticos disponibles en la región, hayan o no sido producidos en Sudamérica. Países exportadores como Venezuela, se inclinan ciertamente por la primera interpretación a través de la cual se limita la importación de recursos energéticos - petróleo en este caso - de mercados externos. En el hipotético caso que el Grupo consiguiese resolver estos elementos básicos y consiguiera consensuar un borrador del Tratado, éste deberá ser aprobado por los doce ministros de energía, por los tres estamentos gubernamentales que componen UNASUR, y, finalmente, ratificado por $2 / 3$ de los parlamentos nacionales, evento que por el momento parece improbable.

El éxito de las negociaciones del Tratado Energético Sudamericano es todavía menos esperable por un factor adicional: la absoluta falta de liderazgo regional en esta materia. Aunque Venezuela sigue siendo el gobierno que impulsa la agenda regional energética, su capacidad de resolver problemas de coordinación y de financiar los costos de la cooperación está en duda, no sólo por su sesgo a favor de gobiernos ideológicamente afines, sino especialmente debido al desplome de los precios del petróleo a partir de 2009 y de la consecutiva crisis económica y política que enfrenta el régimen bolivariano post-Chávez. La falta de liderazgo regional, empero, no es exclusivamente atribuible al gobierno venezolano. El segundo gobierno de
Lula y los dos gobiernos de Dilma Rousseff se han caracterizado por un progresivo repliegue de la política energética brasilera hacia una estrategia nacional desacoplada con la agenda regional. Para decirlo de manera simple: no hay ningún indicador que pueda sugerir que el Estado Brasilero tenga interés en impulsar una agenda de regionalismo energético.

Cuando todavía el área energética era parte de IIRSA, los bancos regionales intentaron impulsar un proyecto de integración regulatoria en el área energética. Los bancos no estaban innovando, sino que buscaban implementar el mandato que los propios gobiernos sudamericanos les habían encomendado en la Cumbre de Guayaquil de 2002. La iniciativa, sin embargo, fue vetada por Brasil en 2005: la integración regional energética era incompatible con la política nacional de energía orientada a la auto-sustentabilidad energética del país, cuyas directrices eran preparadas en el Ministerio de Minas y Energía bajo la conducción de la ministra Rousseff. El descubrimiento de los yacimientos de pre-sal en la cuenca de Santos y la creciente desconfianza de las autoridades energéticas nacionales respecto al regionalismo energético impulsado por Venezuela, son probablemente factores que explican el vuelco nacionalista de la política energética brasilera. Entrevistas con funcionarios de la Empresa de Pesquisa Energéticas (EPE), ente público encargado del diseño de la política energética brasilera, y con el Ministerio de Minas y Energía muestran, por una parte, una falta de conocimiento acerca de lo que se discute al interior del Consejo Energético Sudamericano. Por otra parte, revelan el abandono de la estrategia regionalista/internacionalista asumida por Petrobras a inicios del primer gobierno Lula, y la reorientación hacia una estrategia volcada a la exploración y desarrollo de los nuevos yacimientos (Pimentel, 2013).

Junto con la reorientación hacia "adentro", el Estado brasilero ha emprendido una estrategia de expansión "hacia afuera" que, sin embargo, no pasa por la región sino que 
se enmarca en una política de cooperación Sur-Sur con países del África Sub-Sahariana. Programas tales como ProRenova se gestaron desde el Ministerio de Relaciones Exteriores bajo el segundo gobierno Lula da Silva, con el objetivo político de construir un perfil de liderazgo global (es decir, más allá de Sudamérica) y con el objetivo económico de expandir la industria de los biocombustibles (en la que Brasil es junto con los Estados Unidos el principal actor), y de fomentar la internacionalización de las grandes corporaciones de capital brasilero (tales como Petrobras-Biocombustible, Odebrecht, Guaraní) mediante apoyo finan- ciero del Banco Nacional de Desarrollo Económico y Social (BNDES) y apoyo técnico de la Fundación Getúlio Vargas (Fulquet; Pelfini, 2015). Las capacidades políticas, económicas y de conocimiento-tecnológicos empleados por Brasil en estos programas, contrasta con la falta de interés que las autoridades y los thinktanks brasileros muestran por las actividades del Consejo Energético Sudamericano.

De acuerdo a los mismos funcionarios del Ministerio de Minas y Energías y de EPE, el Estado brasilero prefiere gestionar sus interdependencias energéticas de modo bilateral que de modo multilateral o regional como, por

Cuadro 3 - Acuerdos bilaterales ente Brasil y Estados sudamericanos en el sector energético

\begin{tabular}{|c|c|c|}
\hline Países & Acuerdos & Fecha \\
\hline Brasil-Argentina & $\begin{array}{l}\text { Tratado para el aprovechamiento de recursos hídricos compartidos del } \\
\text { Río Uruguay, Pepiro-Guaçu. }\end{array}$ & 2007 (protocol adicional) \\
\hline Brasil-Uruguay & $\begin{array}{l}\text { Acuerdo de Intercambio Eléctrico } \\
\text { Tratado Bilateral de Integración Energética }\end{array}$ & $\begin{array}{l}\text { 2009, renovado en } 2010 \\
\text { En negociación }\end{array}$ \\
\hline Brasil-Paraguay & $\begin{array}{l}\text { Acuerdo de cooperación en Energía Eólica } \\
\text { Tratado de Itaipú } \\
\text { Plan de Acción para el desarrollo de bicombustibles en Paraguay. }\end{array}$ & $\begin{array}{l}2015 \\
1972 \text { (con modificaciones } \\
2005,2007 \text { y } 2009) \\
2007\end{array}$ \\
\hline Brasil-Bolivia & Acuerdo de suministro de gas & 1996 \\
\hline Brasil-Chile & $\begin{array}{l}\text { Comisión Mixta Permanente en Energía y Minería } \\
\text { Acuerdo de cooperación entre Petrobras y ENAP en biocombustibles, } \\
\text { gas natural y la exploración de la Plataforma del Pacífico. } \\
\text { Acuerdo de cooperación en biocombustibles. }\end{array}$ & $\begin{array}{l}2006 \\
2007 \\
2007 \\
\end{array}$ \\
\hline Brasil-Ecuador & $\begin{array}{l}\text { Memorándum de Entendimiento en cooperación en el sector energético } \\
\text { Acuerdo Complementario al Acuerdo Básico de Cooperación Técnica } \\
\text { "Procesos Agroproductivos para biocombustibles" } \\
\text { Protocolo de Intenciones sobre Cooperación Técnica en el Área de } \\
\text { Energía y de Minas }\end{array}$ & $\begin{array}{l}2007 \\
2007 \\
2007\end{array}$ \\
\hline Brasil-Peru & $\begin{array}{l}\text { Memorándum de Entendimiento para una Comisión mixta sobre } \\
\text { Energía y Minería } \\
\text { Convención sobre Integración Energética } \\
\text { Acuerdo sobre Suministro Eléctrico y Exportación de Excedentes } \\
\text { Programa de desarrollo de cultivos alternativos para biocombustibles. }\end{array}$ & $\begin{array}{l}2006 \\
2008 \\
2010 \text { (archivado por el } \\
\text { gobierno Peruano bajo } \\
\text { decisión del Congreso en } \\
2012 \text { por violar Principios } \\
\text { Constitucionales) } \\
2007\end{array}$ \\
\hline Brasil-Colombia & $\begin{array}{l}\text { Ajustes complementarios al "Acuerdo base de Cooperación Energética" } \\
\text { Cooperación técnica para la exploración del Aceite de Palma y de } \\
\text { Mamona como fuentes de biocombustibles. } \\
\text { Acuerdo de cooperación en biocombustibles }\end{array}$ & $\begin{array}{l}\text { Plan de Energía Rural } \\
\text { (2005, renovado en 2009) } \\
2009 \\
2010\end{array}$ \\
\hline Brazil-Surinam & Proyecto "Apoyo al Desarrollo de Producción de Biocombustibles" & 2009 \\
\hline Brazil-Venezuela & $\begin{array}{l}\text { Programa de Cooperación en la Industria de Etanol } \\
\text { Programa para la interconexión Eléctrica } \\
\text { Ajuste al Acuerdo Base de Cooperación Eléctrica }\end{array}$ & $\begin{array}{l}2005 \\
2008 \\
2009\end{array}$ \\
\hline
\end{tabular}


lo demás, ha procedido históricamente en el caso de la hidroelétrica Itaipú y del gasoducto GasBol (Oxilia, 2009; Seifert, 2011). El Cuadro 3 muestra claramente cómo, desde el 2007, fecha de la histórica Primera Cumbre Energética de Sudamérica en Isla Margarita, Brasil se ha volcado intensivamente a la generación de acuerdos bilaterales de cooperación la mayor parte de ellos en el sector de biocombustibles, dejando de lado la agenda regional.

En ausencia de un Estado que asuma los costos de la integración y que provea los marcos de referencia para orientar la agenda y para resolver los múltiples problemas de coordinación entre gobiernos y entre gobiernos y privados, el Consejo Energético de UNASUR queda a la deriva como un cascarón vacío. El liderazgo podría venir de actores no-estatales como organizaciones regionales, sin embargo ellas han sido dejadas fuera del diseño institucional del Consejo de modo deliberado por parte de los propios gobiernos (Palestini; Agostinis, 2015).

\section{CONCLUSIONES: ¿qué tipo de re- gionalismo?}

A quince años de la primera Cumbre de Brasilia, Sudamérica luce más bien como un espacio fragmentado de economías nacionales orientadas a conseguir su propia seguridad energética, que un espacio regional integrado. No obstante condiciones naturales favorables, intereses nacionales complementarios y la presencia de instituciones regionales, el regionalismo energético sudamericano tiene pocos resultados concretos que exhibir. En efecto los únicos proyectos con un cierto carácter regional que han sido implementados, corresponden a 21 proyectos de interconexión física y que provienen de la cartera de proyectos de IIRSA, hoy administrada por el Consejo de Infraestructura y Planificación de UNASUR (COSIPLAN, 2014), lo que indica que, a pesar de sus bajas capacidades y limitaciones, IIR-
SA puede considerarse como la experiencia más exitosa de cooperación sectorial en el área energética (Palestini; Agostinis, 2014).

En este artículo he argumentado que para ser una política exitosa, el regionalismo energético requiere de instituciones que sean capaces de resolver una doble tensión: primero, la tensión entre la lógica del mercado energético y la lógica política de los gobiernos, y, segundo, la tensión intergubernamental entre preferencias que pueden no ser coincidentes o que pueden ser demasiado de corto plazo como para sostener una política de integración. A partir de los estudios de regionalismo comparado, es posible distinguir al menos tres tipos ideales de "regionalismo energético" según el modo en que esta doble tensión es abordada (Belyi; Makarychev, 2015).

Un primer tipo corresponde a contextos en los cuáles las instituciones regionales y los mercados energéticos son mutuamente estructurantes. Por una parte, las instituciones regionales estructuran - a través de regulación - las estrategias de las empresas en los distintos eslabones de la cadena de valor. Por otra parte, las instituciones son receptivas a las señales de mercado. De acuerdo a Belyi y Makarychev (2015) los casos empíricos que más se aproximarían a este tipo ideal son la Comunidad Europea de Energía que fue formalizada en 2005 y que intenta generar un mercado regional integrado entre los países de la Unión Europea, países del sudeste europeo y de la región del Mar Negro. Otros ejemplos, son las políticas de cooperación e integración energética de los países escandinavos en el mercado eléctrico nórdico, o las políticas de cooperación energética entre el grupo de los países de Visegrad (Polonia, Hungría, Eslovaquia y la República Checa).

El segundo tipo ideal corresponde a contextos regionales donde, si bien existen instituciones regionales, estas están desacopladas de los mercados energéticos. Las instituciones no son capaces de regular o de armonizar la regulación existente, y por tanto son ineficaces 
a la hora de estructurar las estrategias corporativas de los actores de mercado. Las políticas adoptadas a nivel regional son, por ende, ignoradas por los actores privados y públicos a nivel nacional o, en el peor de los casos, pueden ser disruptivas. Un acuerdo binacional, por ejemplo, puede tener efectos marginales a nivel regional, pero también puede ser disruptiva de una estrategia de integración regional, si su adopción no toma en cuenta el resto de las interdependencias energéticas en la región. En este segundo tipo de regionalismo, las instituciones regionales y los mercados energéticos producen sus reglas propias, con lógicas mutuamente indiferentes. Ejemplos empíricos de este segundo tipo son la región del Mar Báltico, así como la región de África Occidental bajo las instituciones de la Economic Community of West African States- ECOWAS (Hancock, 2015).

Finalmente, hay un tercer tipo ideal que corresponde a contextos regionales donde las instituciones regionales son ausentes o extremadamente débiles, y, por tanto, los mercados funcionan de manera autorreferencial. La ausencia de institucionalidad normalmente se traduce en que los Estados más poderosos o mejor dotados de recursos energéticos de la región, imponen sus preferencias sobre el resto de los actores, sean estos gobiernos u actores de mercado. En estos contextos, puede ๑ que existan declaraciones intergubernamenales sobre cooperación, pero en la práctica la ขึ seguridad energética nacional de los actores ํ. más poderosos tiende a inhibir los esfuerzos . cooperativos. Los ejemplos empíricos de este \% tercer tipo son las regiones del Este Europeo y 돈 Asia central en las cuales Rusia juega un pa¿ pel de hegemón manteniendo las instituciones iิ regionales en permanente fragilidad.

¿Dónde ubicar Sudamérica? A la luz de lo argumentado en este artículo, la región parece ubicarse en el segundo tipo ideal. Los gobiernos de la región han emprendido procesos de cooperación e integración energética que se han traducido en la construcción de instituciones con mandatos en el área energética, primero a nivel subregional (MERCOSUR y CAN), y luego a nivel regional, tales como IIRSA, PETROAMERICA y, hoy en día, el Consejo Energético Sudamericano. Sin embargo, estas instituciones han sido incapaces de avanzar en la armonización regulatoria, y están lejos de poder estructurar los mercados energéticos en el sentido de generar incentivos que influyan las estrategias de las empresas energéticas, las cuales siguen una lógica casi autorreferencial - en el caso de las Economías Liberales de Mercado - o están completamente bajo el control de los gobiernos - en el caso de las Economías de Capitalismo de Estado -.

Es poco probable que la región se precipite hacia el tercer tipo ideal. Por una parte, la mayoría de los países de la región cuentan con amplias dotaciones de recursos energéticos lo que reduce las situaciones de dependencia energética o de interdependencias enormemente asimétricas. Por otro lado, no pareciera existir en la región ningún actor capaz de sostener una actitud imperialista o hegemónica e imponer sus preferencias sobre el resto. La política exterior bolivariana de Venezuela fue la experiencia más cercana a una política hegemónica que usó la superabundancia de recursos energéticos y los excedentes del petróleo como incentivo material para "comprar" aliados políticos y favorecer su propia posición negociadora; sin embargo esta política ha demostrado ser extraordinariamente vulnerable a las fluctuaciones del mercado internacional del petróleo. Con la caída de los precios del petróleo a partir de 2009, la economía venezolana ha caído en una profunda recesión agravada por una crisis de gobernabilidad. Las preferencias de Brasil, el otro Estado con superabundancia de recursos energéticos y con capacidad de "liderazgo", están volcadas, por un lado, hacia el mercado y la integración energética interna, y por el otro a la cooperación energética fuera del continente como motor de su sector de biocombustibles. A esto hay que agregar la crisis de legitimidad provocada por prácticas de corrupción en las que se 
encuentran involucrados ejecutivos de Petrobras, políticos y parlamentarios de varios partidos incluyendo al Partido de los Trabajadores (Melo, 2016). En consecuencia, la integración regional no ocupa ningún espacio de relevancia dentro de la política energética nacional brasilera.

El escenario más probable para la región es, por tanto, que permanezca entrampada en el segundo tipo ideal que es una suerte de "zona de indiferencia” (Haas; Schmitter, 1964) en la cual las instituciones regionales - tales como el Consejo Energético Sudamericano sobreviven, pero con una influencia marginal sobre las preferencias tanto de los gobiernos como de los actores de mercado.

Salir de la zona de indiferencia no será fácil. En primer lugar los gobiernos nacionales deberán plantearse nuevamente por qué vale la pena perseguir una política energética regional, en otras palabras: por qué cooperar e integrarse. La respuesta parecía relativamente sencilla en los 2000, cuando todos los países sudamericanos estaban más o menos alineados con una trayectoria liberal de mercado y cuando la gran mayoría de ellos enfrentaba crisis de suministro y déficits de inversión. Ese fue el contexto que llevó a todos los presidentes sudamericanos a aceptar la invitación a Brasilia de F.H. Cardoso. Una vez que las condiciones macroeconómicas mejoraron, a mediados de la década de los 2000, muchos países - partiendo por Brasil mismo - recularon hacia una estrategia nacionalista de autosuficiencia energética. Hoy, en 2015, con la diversidad de regímenes y de modelos de desarrollo, la pregunta por qué cooperar debe ser replanteada: el regionalismo energético requiere de un nuevo consenso intergubernamental que le brinde legitimidad y viabilidad, dos elementos que hoy por hoy no están presente.

Si la respuesta a la pregunta anterior sigue siendo positiva, los gobiernos deberán replantearse cómo cooperar. Este artículo ha mostrado que las tres instituciones regionales - IIRSA, PETROAMERICA y el Consejo Ener- gético Sudamericano - constituyen tres modos distintos de concebir la cooperación y la integración energética. Probablemente la respuesta más adecuada hoy en día sea un modelo similar a IIRSA, pero con capacidades reforzadas. Cuando instituciones regionales son diseñadas estrictamente intergubernamentales corren el riesgo de ser capturadas por los intereses de un actor - PETROAMERICA - o de terminar paralizadas - el Consejo Energético. IIRSA, en cambio, pudo avanzar gracias a una fórmula que combinaba legitimidad intergubernamental - a través de los mandatos de las Cumbres Presidenciales - junto con delegación de competencias a los bancos regionales. Una IIRSA reforzada, requiere no sólo de una mayor delegación de competencias, sino de una delegación más amplia que incluya actores no-estatales como OLADE y CEPAL así como a la propia Secretaría de UNASUR. Requiere además contar con la participación de los actores de mercado. Instituciones regionales, como aquellas que caracterizan el primer tipo ideal, no pueden construirse a la espalda de los intereses y estrategias corporativas de los actores de mercado. Los ministros de energía, deben ser parte de la institucionalidad energética regional y actuar como puente entre la estrategia regional y las estrategias nacionales, pero no puede ser - como en la actual estructura de UNASUR - el único órgano responsable de la estrategia regional.

Recebido para publicação em 15 de outubro de 2015 Aceito em 21 de novembro de 2015

\section{REFERENCIAS}

AALTO, P. States and Markets in Energy Policy. In: BELYI, A.; TALUS, K. eds. States and markets in hydrocarbon sectors. Hampshire: Palgrave Macmillan, p. 40-60, 2015.

ARRIAGADA, G. Petropolitics. A review of energy policy and regional relations, Andean Working Paper, InterAmerican Dialogue. In: Latin America. 2006.

BARRERA-HERNÁNDEZ, L. South American Energy Network Integration: Mission Possible? En Roggenkamp et al. eds. Energy Networks and the Law: Innovative Solutions in Changing Markets. Oxford: Oxford University Press. 2012. 
BECKERT, J. How Do Fields Change? The Interrelations of Institutions, Networks, and Cognition in the Dynamics of Markets. Organization Studies, v. 31, n. 5, p.605-627. 2010.

BELYI, A.; MAKARICHEV, A. Regional Institutions and Energy Markets: Systems, Societies, Communities. In: BELYI, A.; Talus, K. eds. States and Markets in Hydrocarbon Sectors. Hampshire: Palgrave Macmillan, p. 61-82, 2015.

BRUSZT, L.; PALESTINI, S. Regional Development Governance. In: BÖRZEL, T.; RISSE, T. The Oxford Handbook of Comparative Regionalism. Oxford: Oxford University Press. 2016.

CAF. ENERGÍA: una visión sobre los retos y oportunidades en América Latina y el Caribe. Marco Económico y Energético. Documento de Trabajo. CAF- Fundación Bariloche-Universidad de São Paulo. 2013.

COSIPLAN. Cuarto Informe de la Cartera de Proyectos COSIPLAN, BID, CAF, FONPLATA. Montevideo, 2014.

COUTO, L.; PADULA, R. Integração da infraestrutura na América do Sul nos anos 2000: Do regionalismo aberto às perspectivas de mudanças. In: CRIES. El regionalismo "post-liberal" en América Latina y el Caribe: nuevos actores, nuevos temas, nuevos desafíos. Buenos Aires: Argentina. 2012.

FLIGSTEIN, N. Markets as politics: a political-cultural approach to market institutions. American sociological review, v. 61, n. 4, p.656-673. 1996.

FULQUET, G.; PELFINI, A. Brasil as a new international cooperation actor in sub-Saharan Africa: Biofuels at the crossroads between sustainable development and natural resource exploitation. Energy Research $\mathcal{E}$ Social Science, v. 5, p.120-129. 2015

GUSTAFSON, C.; ELLIOT, L. Regional Integration: the political uses of energy policy. In: FONT, M.; RANDALL, L. eds. The Brasilian Sstate: debate and agenda. New York: Lexington Books. 2011.

HAAS, E.B.; SCHMITTER, P.C. Differential economics patterns of political integration: projections about Unity in Latin America. International Organization, v. 18, n. 4, p.705-737. 1964.

HANCOCK, K.J. Energy regionalism and diffusion. In: Africa: how political actors created the ECOWAS Center for Renewable Energy and Energy Efficiency. Energy Research \& Social Science, n. 5, p.105-115. 2015.

KOZULJ, R. Situación y perspectivas del gas natural licuado en América del Sur. CEPAL Serie recursos naturales e ๑ infraestructura. n. 132, p. 1-60. 2008.

워 LUTZ, W. Reformas del Sector Energético, desafíos regulatorios i y desarrollo sustentable en Europa y América Latina. CEPAL ปิ Serie recursos naturales e infraestructura, (26): 1-79. 2002.

今 MALAMUD, A. Jefes de Gobierno y Procesos de Integración: las experiencias de Europa y América Latina.

¿. In: LOMBAERDE, P. De; KOCHI, S.; BRICEÑO-RUIZ, J.

$\infty^{-}$eds. Del regionalismo latinoamericano a la integración interregional. México: Siglo XXI, 2008. pp. 137-162.

MANDUCA, P. La energía en la política sudamericana: \& características de las relaciones entre Brasil y Venezuela. - Revista Mexicana de Ciencias Políticas y Sociales, 57 尺े (216), pp.81-100. 2012.

$\therefore$ MELO, M.A. Crisis and integrity in Brazil. Journal of 을 Democracy, v. 27, n. 2, 2016, p. 50-65.

OLADE. Potencial de Recursos Energéticos y Minerales es en América del Sur. Coincidencias Jurídicas hacia una I Estrategia Regional. Quito: UNASUR-OLADE. 2013.

OLIVEIRA, P.A. De. La Sostenibilidad Energética en Sud $\checkmark$ América El Rol de Brasil. Swiss Agency for Development and Cooperation. 2008.

U OSTROWSKI, W. State Capitalism and the Politics of
Resources. In: BELYI, A.; TALUS, K. eds. States and Markets in Hydrocarbon Sectors. Hampshire: Palgrave Macmillan, 2015. pp. 83-102.

OXILIA, V. Raízes Socioeconômicas da Integração Energética na América do Sul: análise dos projetos Itaipu Binacional, Gasbol e Gasandes. Tesis de Dectorado, Universidad de São Paulo. 2009.

PALESTINI, S. Regímenes de Integración Regional: la construcción institucional de los mercados del sur global. Revista de Sociología, (27): 55-78. 2012.

PALESTINI, S.; AGOSTINIS, G. Constructing regionalism in South America: the cases of sectoral cooperation on transport infrastructure and energy. Journal of International Relations and Development. 2015. Disponible online: http://www.palgrave-journals.com/jird/journal/vaop/ ncurrent/full/jird201515a.html.

Constructing Regionalism in South America: the case of transport infrastructure and energy within UNASUR. EUI Working Papers, RSCAS 2014/73. 2014.

PÉREZ, Fidel; KFURI, R. Alba como integraçao antihegemonica. In: VADELL, T. Javier and Las Casas, ed. Os Novos Rumos do regionalismo e as alternativas politicas na América do Sul. Belo Horizonte: PUC Minas. 2011.

PHILIP, G.; PANIZZA, F. The Triumph of Politics: the return of the left in Venezuela, Bolivia and Ecuador. Cambridge: Polity. 2011.

PIMENTEL, F. O fim da era do petróleo e a mudança do paradigma energético mundial: perspectivas e desafios para a atuação diplomática brasileira. Brasilia: Fundacão Alexandre de Gusmão. 2011.

POLLITT, M.G. The role of Policy in Energy Transitions: Lessons for the Energy Liberalisation Era. Energy Policy, (50), pp.128-137. 2012.

ROZAS, P. Internacionalización y expansión de las empresas eléctricas españolas en América Latina. Santiago de Chile: LOM Ediciones - CEPAL. 2009.

RUBIO, V. The Political Economy of Financial Cooperation in South America: CAF's Continuity, Grouth and its role on Regional Long-Term Development Lending. Paper prepared for the Congress of the Latin American Studies Association (LASA), Washington, DC, May 29- June 1, 2013.

RUDNICK, H.; ZOLEZZI, J. Electric sector deregulation and restructuring in Latin America: lessons to be learnt and possible ways forward. IEE Proceedings, 148(2), p.180. 2001.

SANAHUJA, J.A. Post-Liberal Regionalism in South America: the Case of UNASUR. EUI Working Papers. 2012. SEIFERT, J. Brasilian Energy Policy: New Dynamics between regional integration and self-sufficience. Joint Conference "Whatever Happened to North-South? IPSAECPR, pp.1-26. 2011.

SERBIN, A. Chávez, Venezuela y la reconfiguración política de América Latina y el Caribe. Buenos Aires: Siglo XXI. 2011

TALUS, K. EU Energy Law and Policy. Oxford: Oxford University Press. 2013.

TAVARÉS, J. Da IIRSA ao COSIPLAN: Desafios da Integração Física da América do Sul. Rio de Janeiro: Centro de Estudos de Integração e Desenvolvimento. 2012.

TRILLAS, F.; MONTOYA, M. The Liberalization of Infrastructure in Latin America. In: M. Finger; R. Künneke, eds. International Handbook of Network Industries. Chetenham: Edward Elgar, p. 384-406. 2011. 
LOW INTENSITY ENERGY: governments, markets and institutions in the energy regionalism of South America

\section{Stefano Palestini}

Although it is considered to be a priority area of cooperation, the South American energy regionalism shows signs of stalling, evidenced by fruitless negotiations for a South American Energy Treaty. This article explains the paradox that, despite the political will, favorable structural conditions for integration and the creation of ad hoc regional institutions, energy regionalism failed to implement intergovernmental statements on policies. It argues that none of the established institutions have been able to mediate the dispute between markets and between the preferences of governments. Therefore, a new consensus is necessary to reconsider the need and the ability of the region to advance the energy integration agenda. More generally, the energy sector gives important lessons regarding the future challenges that the regional cooperation will face in public policies in South America.

Keywords: Regionalism. South America. Energy integration. UNASUL-IIRSA
ÉNERGIE DE FAIBLE INTENSITÉ: les gouvernements, les marchés et les institutions au sein du régionalisme énergétique en Amérique du Sud

\section{Stefano Palestini}

Bien qu'il soit considéré comme un domaine prioritaire de coopération, le régionalisme énergétique sud-américain donne des signes d'inertie comme en témoignent les négociations infructueuses pour un Traité Énergétique Sudaméricain. Cet article explique le paradoxe selon lequel, malgré une volonté politique, les conditions structurelles favorables à l'intégration et à la création d'institutions régionales ad hoc, le régionalisme énergétique n’a pas réussi à transformer les déclarations intergouvernementales en politiques d'implantation. On fait valoir qu'aucune des institutions créées n’a été en mesure de résoudre les divergences entre les marchés et les préférences des divers gouvernements. Il faudra donc en arriver à un nouveau consensus pour revoir les besoins et les capacités de la région afin de faire avancer le programme d'intégration énergétique. De manière plus générale, le secteur de l'énergie donne d'importantes leçons quant aux prochains défis que la coopération régionale aura à affronter au niveau des politiques publiques en Amérique du Sud.

Mot-CLÉs: Régionalisme. Amérique du Sud. Intégration énergétique. UNASUL-IIRSA

Stefano Palestini - Sociólogo. Doutor em Ciências Políticas e Sociais. Pesquisador no Departamento de Ciências Sociais e Políticas da Universidade Livre de Berlim, Alemanha. Consultor do Programa de das Nações Unidas para o Desenvolvimento (PNUD-Santiago de Chile) e conferencista em varias Universidades do Chile. 
